\title{
A New Directional Coupler Type Partial Discharge Sensor Installed on the Power Lead of Rotating Machine
}

\author{
Sang-Hwa Yi ${ }^{\dagger}$, Don-Ha Hwang* and Wee Sang Park**
}

\begin{abstract}
For on-line partial discharge (PD) monitoring of rotating machines, a novel sensor is proposed, which can be installed on the power lead inside the terminal box of the machine. The sensor has been designed to have high capacitance, and minimal reflection of measured pulses. As a sensitivity of the sensor, transfer impedance $Z_{t}$ has been measured and compared to conventional coupler-type sensors. A simple method is presented for measuring $Z_{t}$ of coupler sensors, using a vector network analyzer and a practical lead-cable of rotating machine. Through this method, it became possible to measure the $Z_{t}$ of coupler sensors including the installation environment of them. The $Z_{t}$ of the proposed sensor is higher than that of same sized other conventional couplers at frequencies between 30 and $92 \mathrm{MHz}$. Another sensitivity test has been performed using a PD calibrator as a test pulse source. The proposed sensor has higher measured peak voltage than the conventional coupler type sensors when the same charges were input.
\end{abstract}

Keywords: Partial discharge sensors, Directional couplers, Rotating machine, Sensitivity

\section{Introduction}

Nowadays, most of the mechanical problems of rotating machines can be diagnosed by monitoring their current, flux and vibration $[1,2]$. Insulation flaws also can be predicted by monitoring the partial discharges (PDs) generated inside the machines. In order to detect PDs without noise interruption, appropriate PD sensors are necessary.

Various types of sensors have been used to detect PD pulses in rotating machines. The most popular type is a capacitive coupler which encapsulates capacitive sensing components made of epoxy-mica or a ceramic compound $\left(\mathrm{SrTiO}_{3}\right)$ [3-6]. The advantages of this type of sensor are high sensitivity and the noise gating ability by using two sensors in a phase [7]. These sensors have been widely used in hydro generators, turbine generators and motors. Because they are installed on the insulated phase bus, on machine terminals or on cables which are electrically connected to the windings of rotating machines, these sensors should have the same level of reliability as the high-voltage insulating components of the machines. The suitable sensitivity and frequency response of these kinds of sensors are determined by reducing the stray inductance of the sensor or of the installation structure [9].

For the convenience of sensor installation, current

$\dagger$ Corresponding Author: Advanced Medical Device Research Division, Korea Electro-technology Research Institute, Korea (shyi@keri.re.kr)

* HVDC Research Division, Korea Electro-technology Research Institute, Korea. (dhhwang@keri.re.kr)

** Dept. of Electrical Engineering, Pohang University of Science and Technology, Korea. (wsp@postech.ac.kr)

Received: November 19, 2015; Accepted: May 21, 2016 transformers (CTs) also have been used to measure the PD of rotating machines [10]. Though these type of sensors have been used for all kinds of power systems, the representative application of this sensor is insulation diagnosis of cable joints [11, 12]. However, the CTs have low sensitivity at frequencies greater than hundreds megahertz and can be easily interrupted by strong noises that have frequencies less than several megahertz.

Recently, antenna-type UHF PD detectors have been tried to measure the PDs of motors [13-15]. These remotely-mountable sensors can be installed on operating machines without stoppage or interruption. Despite the convenience of these sensors, they have not been used extensively because they have relatively low sensitivity and can be easily affected by outside noises.

Another type of sensor is the directional coupler type which does not directly contact the high voltage part of the machines $[16,17]$. Some of these sensors have been developed for power cable diagnosis [18, 19]. The directional coupler type sensors have both capacitive and inductive components, and the ratio between capacitance and inductance is constant over length: i.e., the sensor is a transmission line structure with constant characteristic impedance $Z_{0}$, and it is usually matched to $50 \Omega$. Some of the couplers have two output ports, and the information obtained from them can be analyzed to distinguish the direction of sensing pulses; others have only one output port and the opposite part is terminated in a $50-\Omega$ load to avoid the reflection of pulses.

Usually, the main signal line of the directional coupler sensors are single thin micro-strip line with a low coupling factor. Accordingly the sensitivities are lower than the capacitive coupler type sensors [20]. The directional coupler 
type sensors are usually installed on the stator winding which is located inside the rotating machine. Thus, in order to install the sensors, the rotor should be disassembled.

This paper suggests a new directional coupler type sensor which can be installed simply on the power leads of highvoltage rotating machines without any electrical connection to their high-voltage components. As a sensitivity of the coupler, transfer impedance has been measured and compared to other conventional couplers. A simple method of measuring transfer impedance is suggested. PD detection experiments are also performed using a PD calibrator source and also using the real PDs from the winding inside a motor. Sensor design and experimental method are described in Section 2, results are given in Section 3, discussion is given in Section 4, and a conclusion is given in Section 5.

\section{Sensor Design and Experimental Method}

\subsection{Sensor design}

The proposed coupler have been designed (Fig. 1) to

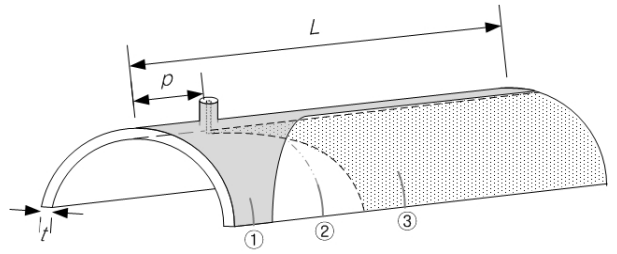

(a)
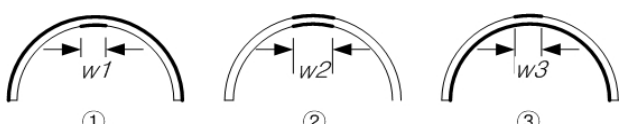

(1)

(b)

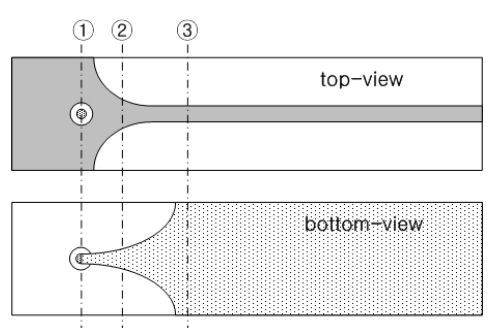

(c)

Fig. 1. Structure of proposed coupler: (a) Configuration of proposed coupler. Dotted face: center conductor; copper on the inner surface. Grey face: ground conductor; copper on the outer surface. (1), (2), (3): positions of sectional view (Fig. 1b). Length $\mathrm{L}=$ $140 \mathrm{~mm}$, thickness $\mathrm{t}=2 \mathrm{~mm}$, port position $\mathrm{p}=20$ $\mathrm{mm}$; (b) Sectional views of proposed coupler. Thick line: conductor, thin line: dielectric. Width of conductor lines: $w 1=4.1 \mathrm{~mm}, \mathrm{w} 2=6.1, \mathrm{w} 3=4.6 \mathrm{~mm}$; (c) Top-view and bottom-view of the coupler. have higher sensitivity than microstrip-line type directional couplers which were generally used for the PD detection of rotating machines or cable joints. The center conductor of the coupler has been designed widely to have high capacitance. The coupler length $L$ is $140 \mathrm{~mm}$ and thickness $t$ is $2 \mathrm{~mm}$. The line-width $w 1$ of the center conductor, the line-width $w 2$ of the ground conductor and port position $p$ have been optimized using electromagnetic field simulation software (CST Microwave studio) to obtain the highest peak voltage. The Gaussian pulse of 0.4-ns fullwidth at half-maximum is used as a source in the simulation. The doubly-tapered structure of center and ground conductor is designed to deliver the measured PD signal with minimized distortion to the port of sensor. As the coupler's body material, a polyvinyl chloride flexible sheet is used which having relative permittivity $\varepsilon_{r}=3.4$. This flexible material helps to reduce the gap between the coupler conductor and the power lead so that the sensor's sensitivity can be increased.

\subsection{Sensitivity testing method}

The sensitivity of the proposed sensor has been compared to those of the conventional coupler type PD sensors. In order to present the coupler sensitivities, the transfer impedance $Z_{t}$ has been evaluated. In general, the parameter $Z_{t}$ had been used to represent the frequency response of current transformers (CT) [18]. However, the experimental environment of $Z_{t}$ is very rigorous because the uncertainty of the test current should be reduced. Therefore, transmission line for the test must be carefully set up to have stable characteristic impedance [22]. Usually, the $Z_{t}$ had been evaluated only for CT type sensors, but not for directional coupler type or capacitive couplers; it was because the latter couplers' sensitivities often vary according to the structure of the installation object, that is the transmission line.

A measuring method has been suggested that uses a real

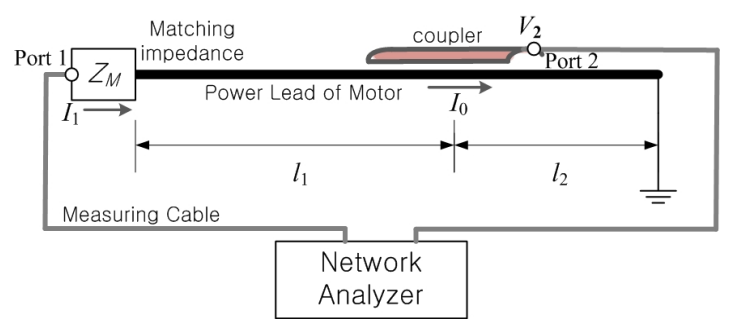

Fig. 2. Experimental circuit for transfer impedance measurement; the circuit is a two-port network: port 1 is connected to the practical power lead (HELUKABLE H07V-K) and port 2 is the coupler's output. $V_{2}$ : measured voltage of coupler output terminal. $I_{0}$ : current of power lead cable. $Z_{M}$ : matching impedance. $l_{1}$ : cable length from port-1 to coupler position. $l_{2}$ : cable length from coupler position to ground termination. 
power lead of motor as a test transmission line as seen in Fig. 2. Using this method, not only the characteristics of the coupler but also that of the power lead structure can be included in measurement results. Most kinds of PD sensors including CT-type, directional coupler type and foil coupler type has been tested.

The transfer impedance of the coupler

$$
Z_{t}=\frac{V_{2}}{I_{0}}=\frac{V_{2}}{I_{1}} C_{I}
$$

has been evaluated from the measurement data of the suggested experimental circuit (Fig. 2), where $C_{I}$ which derived using transmission line theory [21] in the Appendix is the constant factor that converts the current $I_{0}$ at coupler position to the measurable current $I_{1}$ at port 1 .

Generally, the current transformer's $Z_{t}$ provided by commercial manufacturer is measured from the opencircuit voltage of the output terminal. In the experimental circuit of Fig. 2, the open-circuit voltage of coupler is the port-2 voltage $V_{2}$ when the port- 2 current $I_{2}=0$. In this case, $Z_{t}$ (Eq. 1) can be expressed using the impedance parameter $Z_{21}$ of the two-port network in Fig. 2:

$$
Z_{t}=\left.\frac{V_{2}}{I_{1}}\right|_{I_{2}=0} C_{I}=Z_{2 I} C_{I}
$$

The $Z_{21}$ can be evaluated from S-parameters which are measured easily using a network analyzer. The relationship between impedance parameter $Z_{21}$ and S-parameters for two-port network are presented in the Eq. A4 (Appendix). In this way, the transfer impedance in open circuit environment are simply evaluable using network analyzer and Eq. 2.

On the other hand, many PD sensors are matched to $50-\Omega$ transmission lines and $Z_{t}$ values of sensors are provided from the measured voltage at $50 \Omega$ load $\left(Z_{L}\right)$. In this case, the Eq. 2 is not proper. In order to consider the $50-\Omega$ load environment, the experimental two-port network (Fig. 2) should be converted to the equivalent circuit (Fig. 3) with impedance parameters [21]. In this circuit, the transfer impedance

$$
Z_{t}=\frac{Z_{L} Z_{12}}{Z_{11}+Z_{22}-2 Z_{12}+Z_{L}} C_{I}
$$

can be brought by the voltage division rule. The impedance parameters $\left(Z_{11}, Z_{12}, Z_{21}\right.$ and $Z_{22}$ in Eq. 3) can also be evaluated from S-parameters of the same two port network using the equations of Appendix A4 - A7. As a result, the $Z_{t}$ with $50 \Omega$ load is also simply evaluable using Eq. 3. In this method, the $Z_{t}$ values of the proposed coupler and three kinds of conventional PD couplers have been evaluated. The results are provided in the section 3 .

In order to verify the measured $Z_{t}$, an equivalent circuit

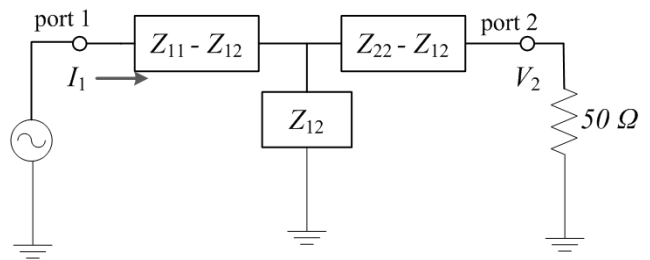

Fig. 3. Equivalent circuit of the experimental two-port network (Fig. 2) expressed using impedance parameters $\left(Z_{11}, Z_{12}\right.$ and $\left.Z_{22}\right)$ of the network.

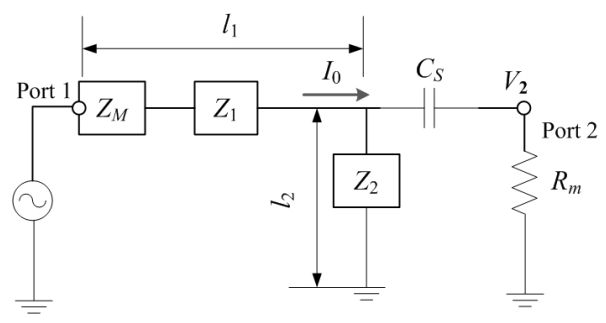

Fig. 4. Equivalent circuit of the experimental circuit in Fig. 2. $R_{m}(\Omega)$ : measuring resistance. $Z_{1}(\Omega)$ : impedance of the motor lead from port-1 to coupler position. $Z_{2}$ $(\Omega)$ : impedance of the motor lead from coupler position to ground termination. $C_{s}(\mathrm{~F})$ : capacitance between the coupler and the motor lead.

of the experimental setup has been presented in Fig. 4 and the theoretical $Z_{t}$ has been calculated using the following Eq. (4).

$$
Z_{t}=\frac{R_{m} Z_{2}}{Z_{2}+R_{m}+\frac{1}{j \omega C_{S}}}
$$

The capacitance of the coupler $C_{s}$ and impedance $\mathrm{Z}_{2}$ of the power lead from coupler position to ground termination in Eq. (4) have been evaluated from the structure of coupler and power lead. The comparison result between experiment and theoretical $Z_{t}$, are presented in section 3 .

The relations between the couplers' practical measured peak voltages and the charges of PD have been evaluated. First, a PD calibrator source (Nihon Keisokuki NPG-2, 10 -ns rising time) has been connected to the terminal of a high voltage induction motor as seen in Fig. 5. The calibrated PD pulses from 1 to $500 \mathrm{pC}$ have been injected to the induction motor. The pulse shapes and spectrum of pulses are presented in Fig. 6. The signals have been measured through several sensors installed on the power lead in the motor terminal box. The proposed coupler, foiltype coupler, strip-line coupler and a CT-type PD sensor have been installed on the same lead cable in the terminal box. A ceramic capacitive coupler $\left(\mathrm{SrTiO}_{3}, 100 \mathrm{pF}\right)$ also has been connected to the terminal of the same phase other sensors were installed on.

The real PD signals generated from the winding of the motor were also measured by several sensors. High voltage 


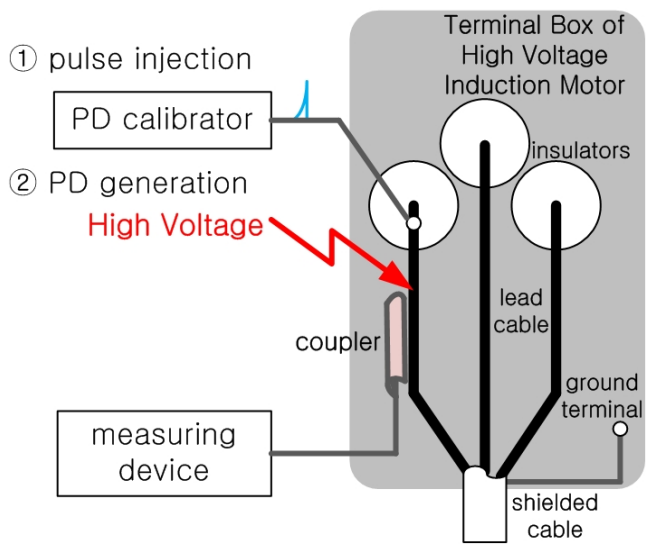

Fig. 5. Setup of PD-pulse sensing experiment. (1) Pulse injection method: inject calibrated PD pulses to the motor power lead using a PD calibrator source. (2) Real PD generation method: supply high voltage $\left(6.6 \mathrm{kV}_{\mathrm{rms}}, 60 \mathrm{~Hz}\right)$ to the power lead and generate the PDs of windings.
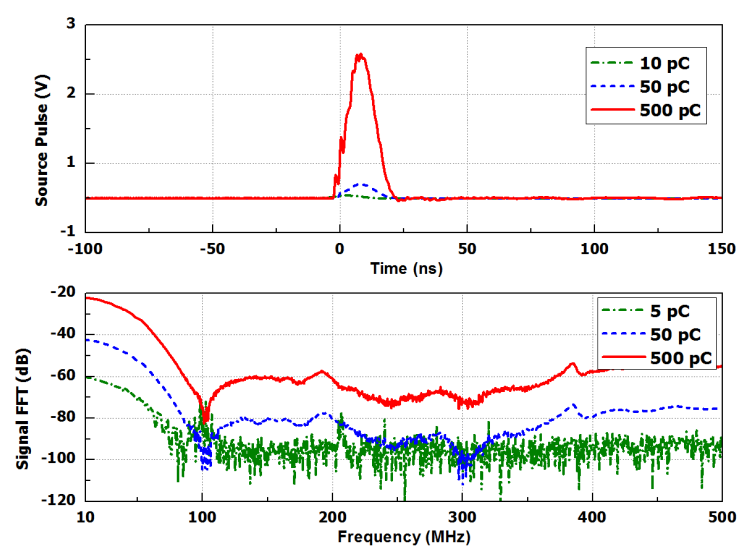

Fig. 6. Calibrated PD pulse signals and their frequency spectrums.

of $6.6 \mathrm{kV}_{\mathrm{rms}}$ and $60 \mathrm{~Hz}$ was applied to the motor winding through the terminal. The PD patterns and pulse shapes of several sensors were measured simultaneously. The proposed coupler, the ceramic capacitive coupler and the CT-type PD sensor were used. The measured PD patterns and pulse shapes are presented in Section 3. The average peak voltage ratios of simultaneously measured pulses are also presented.

\section{Results}

The $Z_{t}$ values of the proposed couplers and conventional couplers have been measured using the experimental circuit of Fig. 2 and evaluated by Eq. (3). A network analyzer (Agilent N5230A) has been used to measure the S-parameters of the network. The results of following sensors are presented in Fig. 7; the proposed coupler having $L=140 \mathrm{~mm}$, a microstrip line coupler having $L=$

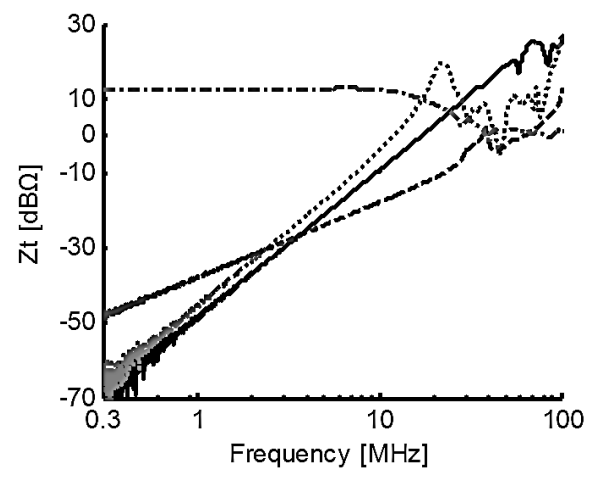

Fig. 7. Measured $Z_{t}$ (50 $\Omega$ load) over frequency for several coupler designs. - : proposed coupler, ...... : foil coupler, ---: strip-line coupler, ---: CT-type PD sensor.

$140 \mathrm{~mm}$, a half-cylindrical foil coupler having $L=140 \mathrm{~mm}$ and a CT-type PD sensor (bandwidth $=150 \mathrm{kHz} \sim 20 \mathrm{MHz}$, nominal $Z_{t}=2.8 \Omega$ ). The microstrip line coupler was composed of a thin center conductor and a ground-plane, which had a $Z_{0}$ of $50 \Omega$. The foil coupler had a simple halfcylindrical conductor, but had neither ground conductor nor tapered structure.

As seen in Fig. 7, $Z_{t}$ of the proposed coupler is higher than any of the other couplers at frequencies $30 \mathrm{MHz} \sim 92$ $\mathrm{MHz}$ and higher than thin microstrip line couplers at frequencies $3.3 \mathrm{MHz} \sim 100 \mathrm{MHz}$. The inductive CT-type PD sensor shows a flat frequency response below $10 \mathrm{MHz}$ whereas the other capacitive couplers show linearly increasing frequency responses. The CT manufacturer's nominal value of $Z_{t}=8.95 \mathrm{~dB} \Omega$ (or $2.8 \Omega$ ) agrees well with the measured value of $Z_{t}=8.91 \mathrm{~dB} \Omega$ at the nominal higher cut-off frequency of $20 \mathrm{MHz}$. The simple foil coupler has 2 $\sim 3 \mathrm{~dB}$ higher $Z_{t}$ than the proposed coupler at frequencies below $15 \mathrm{MHz}$, but has a peak point of resonance at 22 $\mathrm{MHz}$ whereas the proposed coupler has not any resonance point below $100 \mathrm{MHz}$. The strip-line coupler has smaller $Z_{t}$ and smaller slope angle than other couplers which have wide center conductor.

The equivalent circuit of the experimental setup (Fig. 2) has been suggested in Fig. 4 . The theoretical $Z_{t}$ of proposed coupler in the equivalent circuit has been calculated using the Eq. (4). The comparison result between the theoretical $Z_{t}$ and measured $Z_{t}$, are presented in Fig. 8; solid line is measured value, dashed line is calculated value when the motor lead is assumed inductive line with $L=3.6 \mu \mathrm{H} / \mathrm{m}$ and the broken line is calculated value using the measured impedance $Z_{2}$. The results of the equivalent circuit is agreed with both measured values at frequencies $0.7 \mathrm{MHz}$ $60 \mathrm{MHz}$.

The relations between the couplers' practical measured peak voltages and the charges of PD have been evaluated and presented in Fig. 9, when the coupler is installed on a high-voltage motor. The evaluated relations of measured peak voltages versus generated PD charges are linear in all 


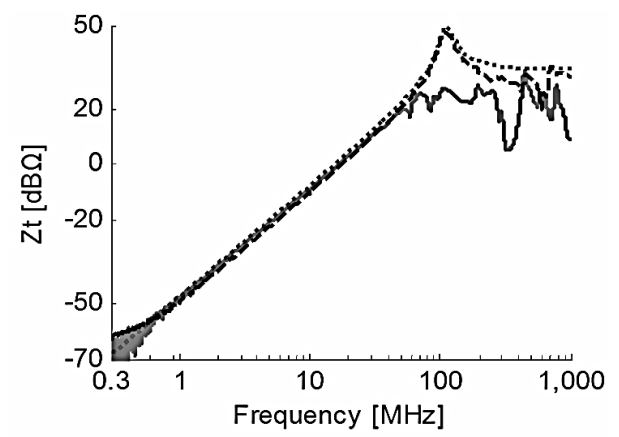

Fig. 8. $Z_{t}$ of proposed coupler $(L=140 \mathrm{~mm})$. - : measured value, ...... : calculated value of equivalent circuit (Fig. 4) when $Z_{2}=j \omega L$ and $L=3.6 \mu \mathrm{H} / \mathrm{m}$, --- : calculated value of equivalent circuit (Fig. 4) using measured $Z_{2}$.

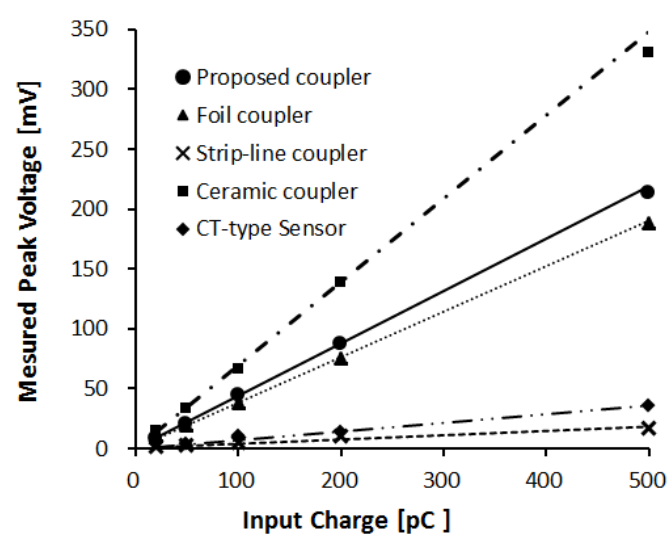

Fig. 9. Measured peak voltage $(y)$ versus PD charge of calibrator $(x)$. proposed coupler: regression $y=$ $0.438 \mathrm{x} ; r^{2}=1$. foil coupler: regression $y=0.381 \mathrm{x} ; r^{2}$ $=1$. strip-line coupler: regression $y=0.0359 x ; r^{2}=$ 0.998. ceramic coupler: regression $y=0.697 \mathrm{x} ; \mathrm{r}^{2}=$ 1. CT-type sensor: regression $y=0.0725 \mathrm{x} ; r^{2}=$ 0.976 .

couplers. It is shown that the proposed coupler can measure 1.15 times higher peak voltage than the foil coupler, 12.2 times higher peak voltage than the strip-line coupler and 6.04 times higher peak voltage than the CTtype PD sensor when the same PD charges are input. The ceramic capacitive coupler of $100 \mathrm{pF}$ can measure highest peak level of all tested sensors

The real PD pulses of motors also have been measured. The patterns (Fig. 10) and pulse shapes (Fig. 11) of several sensors (the proposed coupler, the ceramic capacitive coupler and the CT-type PD sensor) have been measured simultaneously. Because of the limit of channel numbers of the oscilloscope, the proposed sensor and the conventional two sensors were chosen. 3-dB attenuators were used because the amplitude of the measured PD pulse by the proposed coupler and the ceramic capacitive coupler exceeded the range of input amplitude of the oscilloscope. In the pattern result of Fig. 10, the voltage ratios of the

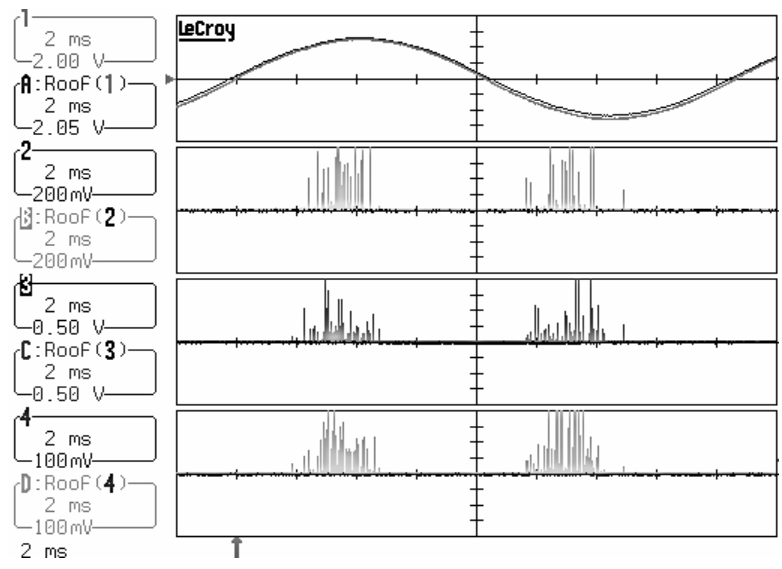

Fig. 10. Measured PD patterns. Channel 1: applied high voltage $(60 \mathrm{~Hz}), 1 / 2000$ probe used. Channel 2: proposed coupler $(\mathrm{L}=140 \mathrm{~mm}), 3-\mathrm{dB}$ attenuator used. Channel 3: ceramic capacitive coupler (100 $\mathrm{pF}$ ), 3-dB attenuator used. Channel 4: CT-type PD sensor. All channel $50 \Omega$ coupling.

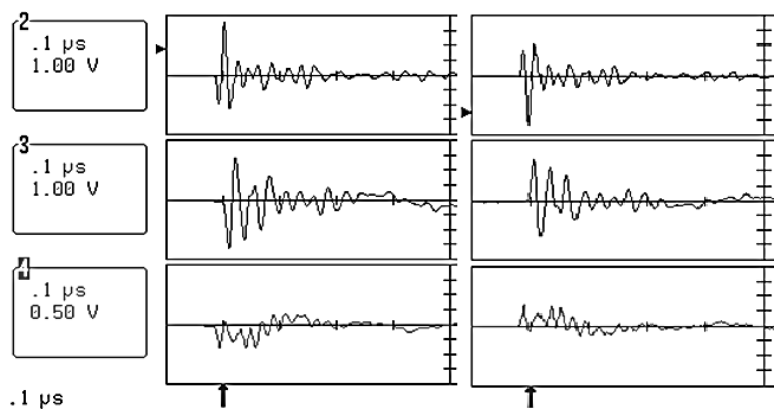

Fig. 11. Measured PD pulse shapes. Left: positive pulses. Right: negative pulses. Channel 2: proposed coupler ( $L=140 \mathrm{~mm}), 3-\mathrm{dB}$ attenuator used. Channel 3: ceramic capacitive coupler $(100 \mathrm{pF}), \quad 3-\mathrm{dB}$ attenuator used. Channel 4: CT-type PD sensor. All channel $50 \Omega$ coupling.

simultaneously measured pulses differed over time; this difference can cause disagreement of PD pattern parameters such as skewness, kurtosis, cross-correlation and asymmetry. As shown in Fig. 11, the proposed coupler could measure positive and negative PD pulses as successfully as other different kinds of PD sensors could. The measured peak voltages of the proposed coupler and the ceramic capacitive coupler were roughly on a level.

In order to compare real PD sensitivities of sensors, peak voltage ratios of simultaneously measured pulses have been evaluated. One hundred pulses have been measured at the same time and the measuring process were repeated four times. Accordingly, four hundreds pulses have been measured and the peak ratios of them were evaluated. The proposed coupler, the foil coupler, the ceramic capacitive coupler and the CT-type PD sensor have been compared. As seen in Fig. 12, the proposed coupler and foil coupler shows higher positive peak level than ceramic coupler 


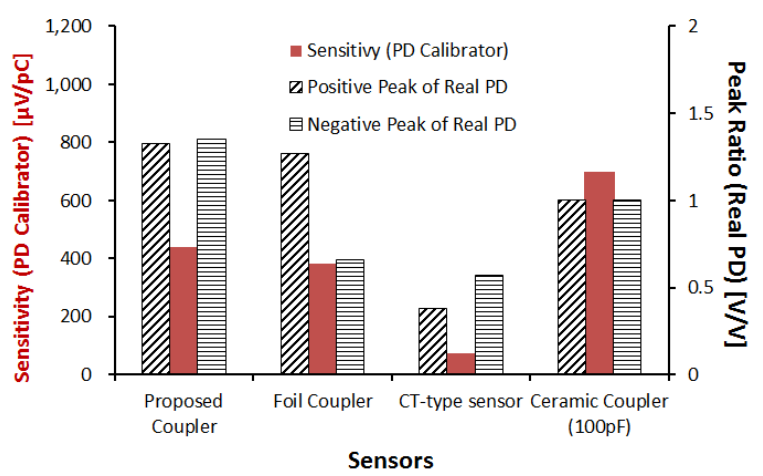

Fig. 12. Positive and negative peak voltage ratios of sensors. Average value of 400 simultaneously measured pulses. Standard deviation: proposed sensor positive 0.181 , negative 0.356 , foil sensor positive 0.104 , negative 0.342 , CT positive 0.058 , negative 0.178 .

which means the PD of solid insulation such as stator winding or end winding contains higher frequency contents than PD calibrator sources in Fig. 6. It seems practical to use proposed coupler to find out insulation defects of stator windings which located near the terminal box. The simple foil coupler has different sensitivities between positive and negative pulses. Negative pulses have higher deviation value than positive pulses have. The proposed and ceramic coupler have similar sensitivities between positive and negative pulses.

\section{Discussion}

The sensitivity $Z_{t}$ values of the proposed coupler and the foil coupler are over $10 \mathrm{~dB}$ above the $Z_{t}$ value of the stripline coupler at frequencies over $8 \mathrm{MHz}$. In general, $Z_{t}$ increases with the capacitance $C_{S}$ between coupler and power lead and $C_{S}$ increases with the conductor area of the coupler. The proposed coupler and the half-cylindrical foil coupler have similarly wide conductors but the strip-line coupler has a narrow conductor. Thus the sensitivity can be increased by increasing the width of the signal line of a coupler. If there were enough space to install, a wider and longer coupler would have higher sensitivity.

The foil sensor shows $3 \mathrm{~dB}$ higher $Z_{t}$ than the proposed coupler at frequencies blow $30 \mathrm{MHz}$. This difference might be due to the small difference of the capacitance of the center conductor. The center conductor area is smaller in the proposed coupler than in the foil coupler because the proposed coupler has the tapered region near the port.

Despite having a bigger conductor than the foil conductor, the proposed coupler shows higher $Z_{t}$ than the foil coupler at frequencies over $20 \mathrm{MHz}$, because the tapered structure of the proposed coupler can deliver the coupled signal efficiently to its port. Basically, $Z_{t}$ is a function of the lead structure and coupler dimensions; increasing the coupler dimensions allow increasing the power of signals induced, but to achieve combination of large coupling and sufficient matching, coupler structure must be carefully optimized. The simple capacitor-type foil sensor does not have the tapered structure for matching so this sensor shows peak resonance at $22 \mathrm{MHz}$ in Fig. 7. The occurrence of a single peak point of $Z_{t}$ is caused by insufficient input current $I_{o}$ at the resonance frequency. This high single peak does not mean high sensitivity, but rather distortion of the sensing signal.

In all couplers, $Z_{t}$ increased with the frequency until $22 \mathrm{MHz}$. This is because at low frequency, the couplers act like simple capacitors connected to the motor lead. However, at high frequency, a coupler is not a simple capacitor, so inductive components of the coupler structure make $Z_{t}$ saturate. Thus, designing the shape of a coupler conductor which affects the inductive components may be also important. However, predicting the characteristic of a coupler is very difficult because both inductive and other parasitic components affect the coupler's sensitivity. This is why the theoretical $Z_{t}$ of the proposed coupler did not match the measured $Z_{t}$ at frequencies over $60 \mathrm{MHz}$. However, our evaluation method was useful enough to roughly predict the sensitivities of the couplers.

The signals from sensors have been measured and the peak voltages have been compared when the same charges from PD calibrators are input. Because the frequency spectrum of every real PD pulse is not unique, an experiment using a calibrator source can be useful to test coupler sensitivity. Actually in Fig. 10, the voltage ratios between the simultaneously measured pulses differ among the times of measurement. If the measured pulses came from the same PD source with a fixed frequency spectrum and came through the same transmission path, the voltage ratios would not have changed. In the field, not only the discharge types such as slot discharge, internal discharge and end-winding discharge but also the frequency characteristics of the PD transmission paths are various. Therefore objective evaluation of sensitivity must be performed before applying PD sensors in the field.

\section{Conclusion}

A coupler is developed for the detection of PD in rotating machines; the proposed coupler can be installed easily on the power lead of the rotating machine without any electrical connection to its high-voltage part.

In order to evaluate the sensitivity of the coupler, the transfer impedance $Z_{t}$ was measured and compared to other conventional couplers. A new method of measuring $Z_{t}$ using the real lead-cable as a transmission line is suggested. $Z_{t}$ of the directional coupler and capacitive foil coupler can also be measured with this method. The measured and modeled $Z_{t}$ values of the proposed coupler agrees well at frequencies below $60 \mathrm{MHz}$ and are higher than the $Z_{t}$ of 
other conventional couplers for the frequency range between 30 and $92 \mathrm{MHz}$.

The proposed coupler can measure 1.15 times higher peak voltage than the foil coupler, 12.2 times higher peak voltages than the strip-line coupler and 6.04 times higher peak voltage than the CT-type PD sensor when the same PD charges are input. Through this measuring experiment using a PD calibrator source, the linearity and reasonable sensitivity of the proposed coupler are verified. The real PD pulses from the winding of the high-voltage induction motor are measured successfully by the coupler.

The suggested coupler might be an effective sensor to detect PD of the rotating machines because it is sufficiently sensitive, convenient to install and relatively safe.

\section{Appendix}

In the experimental circuit for the transfer impedance measurement (Fig. 2), the current $I_{0}$ of the power lead at the coupler position and the current $I_{1}$ at port 1 is [21]:

$$
\begin{gathered}
\boldsymbol{I}_{0}=\boldsymbol{I}_{s}^{+}\left(\boldsymbol{e}^{\gamma l_{2}}-\Gamma \boldsymbol{e}^{-\ell_{2}}\right) \\
\boldsymbol{I}_{\boldsymbol{I}}=\boldsymbol{I}_{s}^{+}\left(\boldsymbol{e}^{\gamma\left(\boldsymbol{l}_{1}+l_{2}\right)}-\Gamma \boldsymbol{e}^{-\gamma\left(l_{1}+l_{2}\right)}\right)
\end{gathered}
$$

where, $\gamma$ is the propagation constant of a power lead which terminates in a shorted circuit, $\Gamma=-1$ is the voltage reflection coefficient at the short termination, $I_{s}^{+}$is the current traveling to the right direction at the short termination. Accordingly, the converting factor

$$
C_{I}=\frac{I_{1}}{I_{0}}=\frac{e^{-\gamma\left(l_{1}+l_{2}\right)}+e^{\gamma\left(l_{1}+l_{2}\right)}}{e^{-\gamma_{1} l_{2}}+e^{\gamma l_{2}}}
$$

is derived from Eqs. A1 and A2.

The relationships between impedance parameters and Sparameters for a two-port network are shown below [19].

$$
\begin{aligned}
& Z_{11}=Z_{o} \frac{\left(1+S_{11}\right)\left(1-S_{22}\right)+S_{12} S_{21}}{\left(1-S_{11}\right)\left(1-S_{22}\right)-S_{12} S_{21}} \\
& Z_{21}=Z_{o} \frac{2 S_{21}}{\left(1-S_{11}\right)\left(1-S_{22}\right)-S_{12} S_{21}} \\
& Z_{12}=Z_{o} \frac{2 S_{12}}{\left(1-S_{11}\right)\left(1-S_{22}\right)-S_{12} S_{21}} \\
& Z_{22}=Z_{o} \frac{\left(1-S_{11}\right)\left(1+S_{22}\right)+S_{12} S_{21}}{\left(1-S_{11}\right)\left(1-S_{22}\right)-S_{12} S_{21}}
\end{aligned}
$$

\section{References}

[1] A. H. Bonnet and G. C. Soukup, "Cause and analysis of stator and rotor failures in three-phase squirrelcage induction motors," IEEE Trans. Ind. Applicat., vol. 28, pp. 921-937, July/Aug. 1992.

[2] D. G. Dorrell, W. T. Thomson, and S. Roach, "Analysis of airgap flux, current, and vibration signals as a function of the combination of static and dynamic airgap eccentricity in three-phase induction motors," IEEE Trans. Ind. Applicat., vol. 33, pp. 2434, Jan./Feb. 1997.

[3] T. E. Goodeve, G. C. Stone and L. Macomber, "Experience with compact epoxy-mica capacitors for rotating machine partial discharge detection," Electrical Electronics Insul. Conf., 1995, and Electrical Manufacturing \& Coil Winding Conf. Proceedings, pp. 685-689, 1995.

[4] J. P. Zondervan, E. Gulski and J. J. Smit, "Fundamental aspects of PD patterns of on-line measurements on turbogenerators," IEEE Trans. Dielectr. Electr. Insul. vol. 7, no. 1, pp. 59-70, Feb. 2000.

[5] Y.-J. Kim, D.-H. Hwang, D.-Y. Park, and S.-O. Song, "Novel On-Line Partial Discharge Monitoring System for Generator/Motor Stator Insulations," Proc. 5th Int. Conf. Electrical Machines and Systems, pp. 330-334, 2001.

[6] J.-H. Sun, Y.-W. Youn, D.-H. Hwang and D.-S. Kang, "Consideration on the Long-term Reliability of Online Partial Discharge Ceramic Sensor for Thermal Power Generators and its Demonstration in the Field," Journal of Electrical Engineering \& Technology, vol. 7, no. 1, pp. 103-108. 2012.

[7] H. Zhu, V, Green. M. Sasic and S. Halliburton, "Increased sensitivity of capacitive couplers for inservice PD measurement in rotating machines," IEEE Trans. Energy Convers, vol. 14 , Issue 4, pp. 11841192, 1999.

[8] M. Kurtz and J.F. Lyles, "Generator Insulation Diagnostic Testing," IEEE Trans. Power App. Syst., vol. PAS-98, pp.1596-1603, Sep/Oct 1979.

[9] M. Muhr and R. Woschitz, "Partial discharge diagnostic," Properties and Applications of Dielectric Materials, Proceedings of the 6th Int. Conf. vol. 1, pp. 223-226, 2000.

[10] J. E. Timperley, "Incipient Fault Identification Through Neutral RF Monitoring of Large Rotating Machines", IEEE Trans. Power Syst. and App., vol. PAS-102, pp. 693-698, Mar. 1983.

[11] K. Mallikarjunappa and M. C. Ratra, "Detection of partial discharges in power capacitors using high frequency current transformers," Electrical Insulation and Dielectric Phenomena Conf., pp. 379-384, 1990.

[12] U. Schichler, "A Sensitive Method for On-Site Partial Discharge Detection on XLPE Cable Joints", Proc. of the 5th Int. Conf. on Properties and Applications of Dielectric Materials, vol. 2, pp. 1099-1102, 1997.

[13] K.-S. Lwin, K.-J. Lim, D.-H. Shin, N.-J. Park, D.-H. Park, K. B. Cho, and H.-D. Kim, "Off-Line PD 
diagnosis for stator winding of Rotating Machines using UWB sensors", Int. Conf. Condition Monitoring and Diagnosis, Beijing, China, pp. 155-158, 2008.

[14] H. Muto, Y. Kaneda, H. Aoki, and O. Hamammoto, "On-line PD monitoring system for rotating machines using narrow band detection of EM wave in $\mathrm{GHz}$ range", Int. Conf. Condition Monitoring and Diagnosis, Beijing, China, pp. 1093-1096, 2008.

[15] Y. Kaneda, M. Yoshimura, H. Muto, E. Iwanaga, and O. Hamamoto, "Monitoring system of partial discharge for high-voltage motors using $\mathrm{GHz}$ electromagnetic wave detection", Annual National Convention of IEE of Japan, 2-029, 2005.

[16] H. G. Sedding, R. R. Campbell, G. C. Stone and G. S. Klempner, "A New Sensor for Detecting Partial Discharges in Operating Turbine Generators," IEEE Trans. Energy Convers., vol. 6, no. 4, pp.700-706, Dec. 1991.

[17] S-H Yi, J-H Sun, D-H Hwang and D-S Kang, "Research on PD Sensor Design for Stator Winding Diagnosis of Turbine Generators", Int. Power Eng. Conf., pp. 1777-1780, 2007.

[18] D. Pommerenke, T. Strehl, R. Heinrich, W. Kalkner, F. Schmidt and W. Weissenberg, "Discrimination between internal PD and other pulses using directional coupling sensors on HV cable systems", IEEE Trans. Dielectr. Electr. Insul. vol. 6, pp. 814-824, 1999.

[19] P. Craatz, R. Plath, R. Heinrich and W. Kalkner, "Sensitive on-site PD measurement and location using directional coupler sensors in $110 \mathrm{kV}$ prefabricated joints," High Voltage Engineering, 1999. Eleventh Int. Symp. vol. 5, pp. 317 - 321, 1999.

[20] CIGRE Working group D1.33 "Guidelines for Unconventional Partial Discharge Measurements," Cigre Technical Brochure, DEC, 2010.

[21] David M. Pozar, "Microwave Engineering", Third Edition, John Wiley \& Sons Inc., pp. 68-69, 193, 211, 2005.

[22] D. Pommerenke, R. Chudru, and S. Chandra, "A new test setup and method for the calibration of current clamps," IEEE Trans. Electromagn. Compt., vol. 47, no. 2, May 2005.

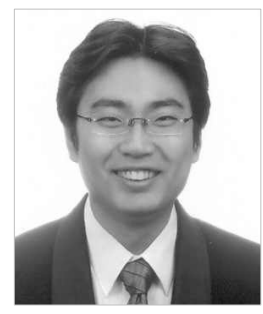

Sang-Hwa Yi He received B.S. degree in Electronic Engineering from Korea University, Seoul, Korea in 2001; and he received an M.S. degree and a Ph.D. degree in Microwave Engineering from Pohang University of Science and Technology (POSTECH) in 2003 and 2016, respectively. Since joining the Korea Electro-technology Research Institute (KERI) in 2003, he has been developed various online PD diagnosing systems including sensors for GIS, transformers and rotating machines. His research interests include diagnosis of power facilities using microwave engineering. Currently, he is a senior researcher in KERI.

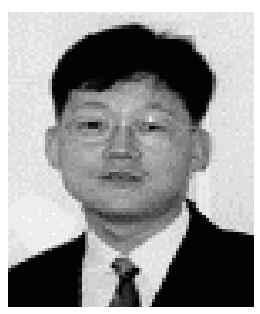

Don-Ha Hwang He received the B.S., M.S. and Ph.D. degrees in Electrical Engineering from Yeungnam University, Kyongsan, Kyungbuk, Korea, in 1991, 1993 and 2003, respectively. Since 1993, he has been with the Power Facility Diagnosis Research Center, KERI, Korea, as a principal researcher. His main research interests are design, analysis, monitoring, and diagnosis of rotating machines.

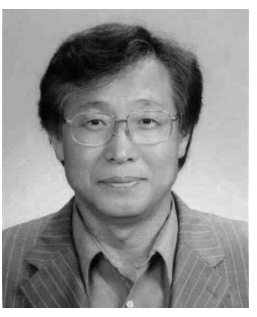

Wee Sang Park He received a B.S. degree in electronic engineering from Seoul National University, Korea, in 1974; and he received an M.S. degree and a Ph.D. degree in electrical engineering from the University of Wisconsin-Madison in 1982 and 1986, respectively. From 1986 to 1988, he taught at Wichita State University. He joined the Pohang University of Science and Technology, Korea in 1988, and is currently a full Professor in the Department of Electrical Engineering. In 1997 he was on a sabbatical leave at the University of Utah. Since 1995, he has been director of the Antenna Laboratory at POSTECH, and has conducted research on the subjects of small antennas, metamaterial antennas, dielectric constant measurement, frequency selective surface, microstrip array, wireless power transfer, RFID, and helical lines. Professor Park is a member of the IEEE MTT and AP societies. 